\title{
Acute effect of coffee consumption on urinary proteome in young healthy individuals
}

\begin{abstract}
Urine, which is not regulated by homeostatic mechanisms, is more likely to reflect early changes and be a source for early biomarker discovery. But urine can be affected by various factors. Coffee is widely consumed in daily life. It may have effects on urinary biomarker discovery and validation in clinical samples. An evaluation of acute coffee effect on urinary biomarker discoveries is needed. In this study, ten healthy volunteers consumed 3 bags of instant coffee in one day. Urine was collected before and after coffee intake. All urine samples were analyzed by liquid chromatography tandem mass spectrometry. After normalization of spectra, $1418 \pm 148$ proteins were identified, indicating that the sympathetic nervous system exerts a significant impact on urine proteome. Eleven proteins were found significantly changed in all ten urine samples after coffee intake and one differential protein was previously annotated as urinary candidate biomarker. Coffee is one of the most consumed beverages worldwide. Thus, when using clinical samples, the effect of coffee should be taken into consideration.
\end{abstract}

Volume 5 Issue 2 - 2017

\author{
Yanying Ni,' Fanshuang Zhang,' Manxia An,' \\ Wei Yin, 'Youhe Gao',2 \\ 'Department of Pathophysiology, Institute of Basic Medical \\ Sciences, Chinese Academy of Medical Sciences, China \\ ${ }^{2}$ Department of Biochemistry and Molecular Biology, Beijing \\ Normal University, China
}

\begin{abstract}
Correspondence: Youhe Gao, Department of Biochemistry and Molecular Biology, Beijing Normal University, Gene Engineering and Biotechnology Beijing Key Laboratory, Beijing, 100875, PR. of China, Tel 861058804382, Email gaoyouhe@bnu.edu.cn
\end{abstract}

Received: February 19, 2017 | Published: March 03, 2017

Keywords: coffee intake, confounding factors, urinary proteome

Abbreviations: LC-MS/MS, liquid chromatography tandem mass spectrometry; SBP, systolic blood pressure; DBP, diastolic blood pressure; $\mathrm{HR}$, heart rate; $\mathrm{Na}$, sodium; $\mathrm{K}$, potassium; $\mathrm{Cl}$, chorion; $\mathrm{Cr}$, creatinine; SDS-PAGE, sodium dodecyl sulfate polyacrylamide gel electrophoresis; DTT, dithiothreitol; IAA, iodoacetamide

\section{Introduction}

Biomarkers are measurable changes associated with a physiological or pathophysiological process. ${ }^{1}$ Unlike cerebrospinalfluid or blood, which to remove changes, urine accumulates and reflects many changes of the whole body. ${ }^{2}$ Thus, urine has potential to be a better biomarker source. In addition to diseases of urinary systems, the valuable information from other system diseases can be found in urine too.

As a sensitive body fluid, urine will be affected by various factors, ${ }^{3,4}$ such as diet, drugs, hormone status or other physiological conditions. ${ }^{5}$ The differential proteins caused by confounding factors may interfere some valuable urinary information discovery. It is difficult to accurately determine whether potential biomarkers are associated with any particular pathophysiological condition or confounding factors, especially in clinical urine samples.

Coffee is one of the most consumed beverages worldwide, which is also rich in bioactive compounds. ${ }^{6}$ Some studies suggested that coffee of both caffeinated and decaffeinated drinking plays an important role in the prevention of some diseases. ${ }^{7}$ However, little is known regarding the influence of coffee on the urinary proteome even though coffee's beneficial effects have been studied extensively. A better understanding of its effects on the urinary proteome is necessary for urinary biomarker research.

In this study, the short-term effects of coffee on the urinary proteome were investigated using label-free quantitative proteomics. Urine samples were collected before and after coffee consumption. Urinary proteins were identified using liquid chromatography tandem mass spectrometry (LC-MS/MS) (Figure 1).

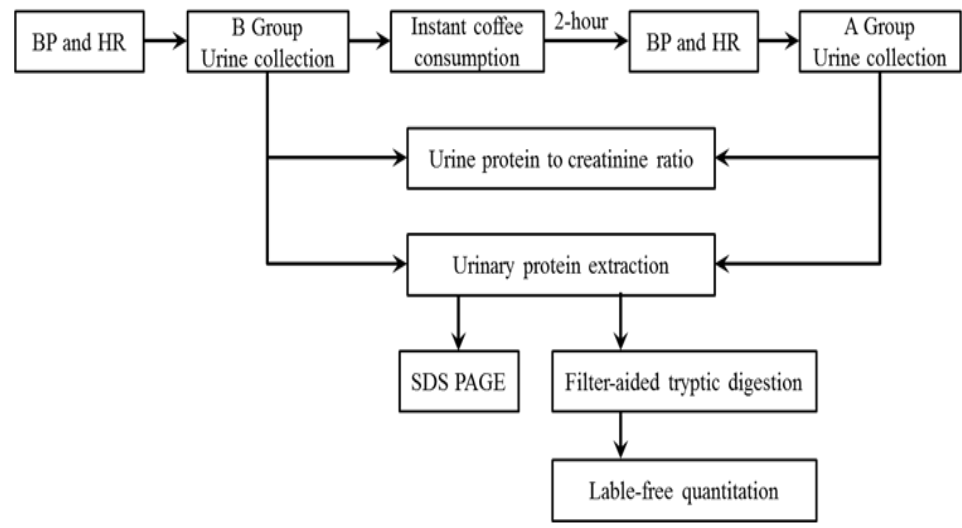

Figure I Workflow of effect of acute coffee consumption on urine proteome.

Urine was collected before and after coffee intake from healthy volunteers. The BP and HR were measured before urine collection. The urinary proteins were profiled by label-free LC-MS/MS. 


\section{Materials and methods}

\section{Ethics statement}

Ten healthy volunteers aged 25-30years, including 5 men and 5 women, were recruited from Peking Union Medical College, Institute of Basic Medical Sciences, Chinese Academy of Medical Sciences, Beijing, China, during August 2016. Informed consents of all volunteers were obtained before the study. The study was approved by the Medical Ethics Committee of Peking Union Medical College (\#046-2016).

\section{Sample collection and subject characteristics}

All volunteers are not habitual coffee drinker and drank three bags of instant coffee (Nescafé Classic, Nestlé, 15 grams) in this study. Urine samples were collected from ten volunteers before and two hours after coffee intake, respectively.

Before urine collecting, systolic blood pressure (SBP), diastolic blood pressure (DBP) and heart rate (HR) were measured using an automatic arm sphygmomanometer (HEM-7052, OMRON, China) 3 times, with a $1 \mathrm{~min}$ interval between each measurement. Urinary protein, creatinine concentrations and ions in 20 samples were measured at the Peking Union Medical College Hospital.

\section{Urinary protein preparation}

Urine was centrifuged at $2000^{\prime} \mathrm{g}$ and $12000^{\prime} \mathrm{g}$ for 30 minutes at $4^{\circ} \mathrm{C}$ respectively to remove precipitates. Urinary proteins were extracted by ethanol and protein precipitation were dissolved in lysis buffer (8M urea, $2 \mathrm{M}$ thiourea, $25 \mathrm{mM}$ dithiothreitol and 50mM Tris). ${ }^{9}$

Samples were divided into two parts. Some proteins were separated by SDS-PAGE. Some urinary proteins of each samples were digested by trypsin (Trypsin Gold, Mass Spec Grade, Promega, Fitchburg, WI, USA) in $10 \mathrm{kD}$ filter units (Pall, Port Washington, NY, USA). ${ }^{10}$ UA (8 $\mathrm{M}$ urea in $0.1 \mathrm{M}$ Tris- $\mathrm{HCl}, \mathrm{pH} 8.5$ ) was firstly added to wash the impurities, and $\mathrm{NH} 4 \mathrm{HCO} 3$ was added to wash the remaining UA in the protein solution. After denatured by DDT and alkylated by IAA, the urinary proteins were digested by trypsin with ratio (Enzyme to protein is $1: 50$ ).

The collected peptide mixtures were desalted by Oasis HLB cartridges (Waters, Milford, MA) and dried by vacuum evaporation (Thermo Fisher Scientific, Bremen, and Germany).

\section{Urinary proteins analysis by LC-MS/MS}

The lyophilized peptides were acidified with $0.1 \%$ formic acid, and subjected to a reversed-phase micro-capillary column. The binding peptides were eluted by a gradient of buffer B $(0.1 \%$ formic acid, $99.9 \%$ acetonitrile; flow rate, $0.3 \mathrm{~L} / \mathrm{min}$ ) for $100 \mathrm{~min}$ and were analyzed using Thermo Orbitrap Fusion Lumos (Thermo Fisher Scientific, Bremen, and Germany).

\section{Label-free quantitation}

The MS/MS data were analyzed by Progenesis software. Database was set as Uniprot_human database (Homo sapiens 84888 sequences; $34,737,292$ residues); the precursor mass tolerance and the fragment tolerance were set $0.05 \mathrm{Da}$; fixed modification was set carbamidomethylation of cysteines; variable modification was set oxidation of methionine and protein N-terminal acetylation. After normalization, the relative abundance was used to analyze differential expressed proteins.

\section{Statistical analysis}

Comparisons between before and after coffee intake were followed by Tukey's multiple comparison tests. P values of less than 0.05 were considered statistically significant. Statistical analysis was performed by the Statistical Package for Social Studies version 16.0.

\section{Results and discussion}

\section{Transient changes on BP, HR and urine ion after consumption of coffee}

The results of the present study showed that acute instant coffee consumption causes a short time decrease in heart rate but does not significantly affect the BP (Figure 2). The concentration of urinary sodium $(\mathrm{Na})$, potassium $(\mathrm{K})$, chorion $(\mathrm{Cl})$ and creatinine $(\mathrm{Cr})$ were found decrease in all urine samples after coffee intake (Figure 3), which may be caused by urine volume increased. The concentrations of urinary ions were measured in urine samples which were collected about two hours after coffee intake instead the total urine output of a specific time frame.
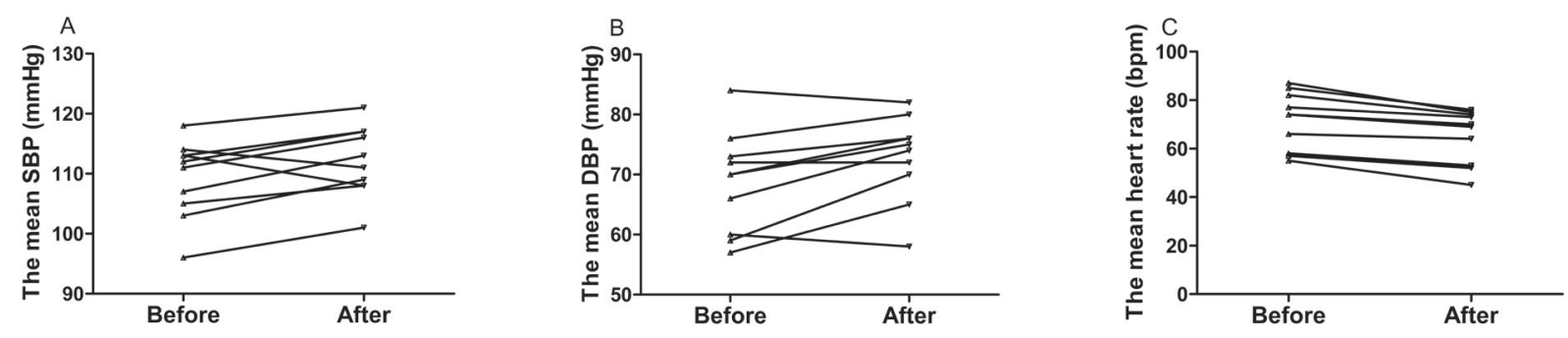

Figure 2 Effect of instant coffee on SBP, DBP and HR before and after coffee intake. A. SBP increased in eight volunteers. B. DBP increased in seven volunteers. C.All volunteers heart rate decreased after acute coffee intake.'

\section{SDS-PAGE analysis of the urinary proteins}

The urinary proteins were analyzed using SDS-PAGE (Figure 4). Compared with urinary proteins before coffee intake, the urinary patterns after coffee intake did not show any significant changes.

\section{Urinary proteomics analysis of acute Coffee intake on} the human urinary proteome

The urinary samples from ten healthy human volunteers were analyzed using Thermo Orbitrap Fusion Lumos. Each sample was analyzed twice to provide technical replicates (Table S1). After normalization of spectra, $1418 \pm 148$ proteins were identified (Table $\mathrm{S} 2)$. According to the standard $\left(\mathrm{p}<0.05\right.$, fold change $£ 0.67$ or $\left.{ }^{3} 1.5\right)$, eleven proteins were found to be significantly altered after coffee intake (Table 1), suggesting that acute coffee consumption can affect the urinary proteome. Among these proteins, six proteins showed increased in all ten urine samples after coffee intake and five proteins showed decreased. All differential proteins were searched against the 
Urinary Protein Biomarker Database, ${ }^{11}$ one protein has been reported as candidate biomarkers of bladder cancer. ${ }^{12}$

Eleven differentially expressed proteins were analyzed using the IPA tool to assess their association with important biological functions. The canonical pathway analysis demonstrated that protein changes were highly associated with Hepatic fibrosis/ hepatic stellate cell activation, Glycolysis and Inhibition of matrix metalloproteases
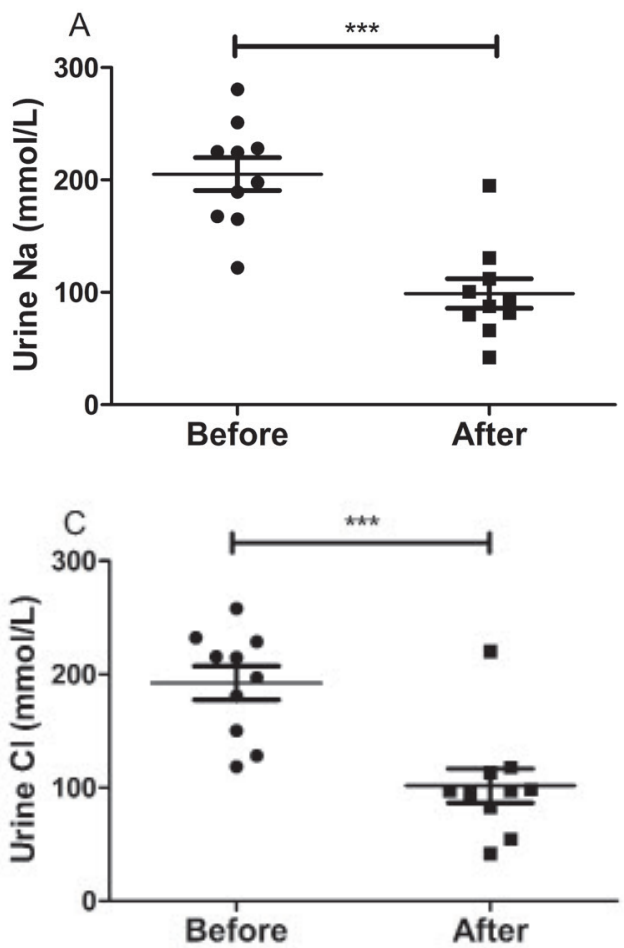

Figure 3 Effect of instant coffee on urinary ions.

A. The urinary $\mathrm{Na}$; $\mathrm{B}$. The urinary $\mathrm{K}$; $\mathrm{C}$ :The urinary $\mathrm{Cl}$; D.The urinary $\mathrm{Cr}$.
signaling(Figure 5A). Metalloproteinase inhibitor 2 (TIMP2), involved in renal tubular epithelial cell cycle arrest in acute kidney injury, play a significant role in several pathways, ${ }^{13}$ such as Hepatic fibrosis/ hepatic stellate cell activation, Inhibition of matrix metalloproteases signaling and glioma invasiveness signaling. 14-3-3 protein gamma (YWHAG) also involved in several canonical pathways, such as cell cycle: G2/M DNA damage checkpoint regulation, ERK5 signaling, Myc mediated apoptosis signaling and HIPPO signaling.
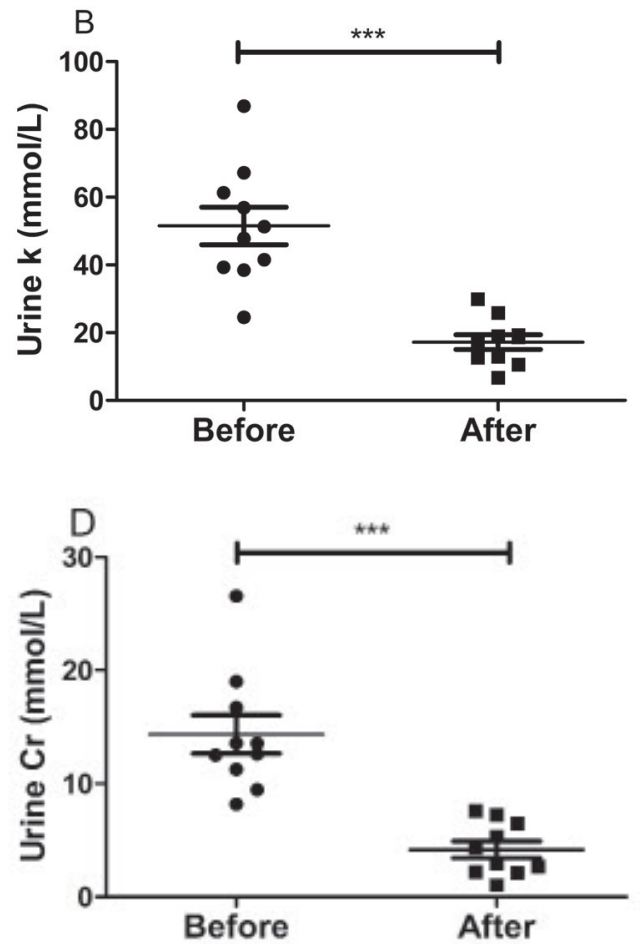

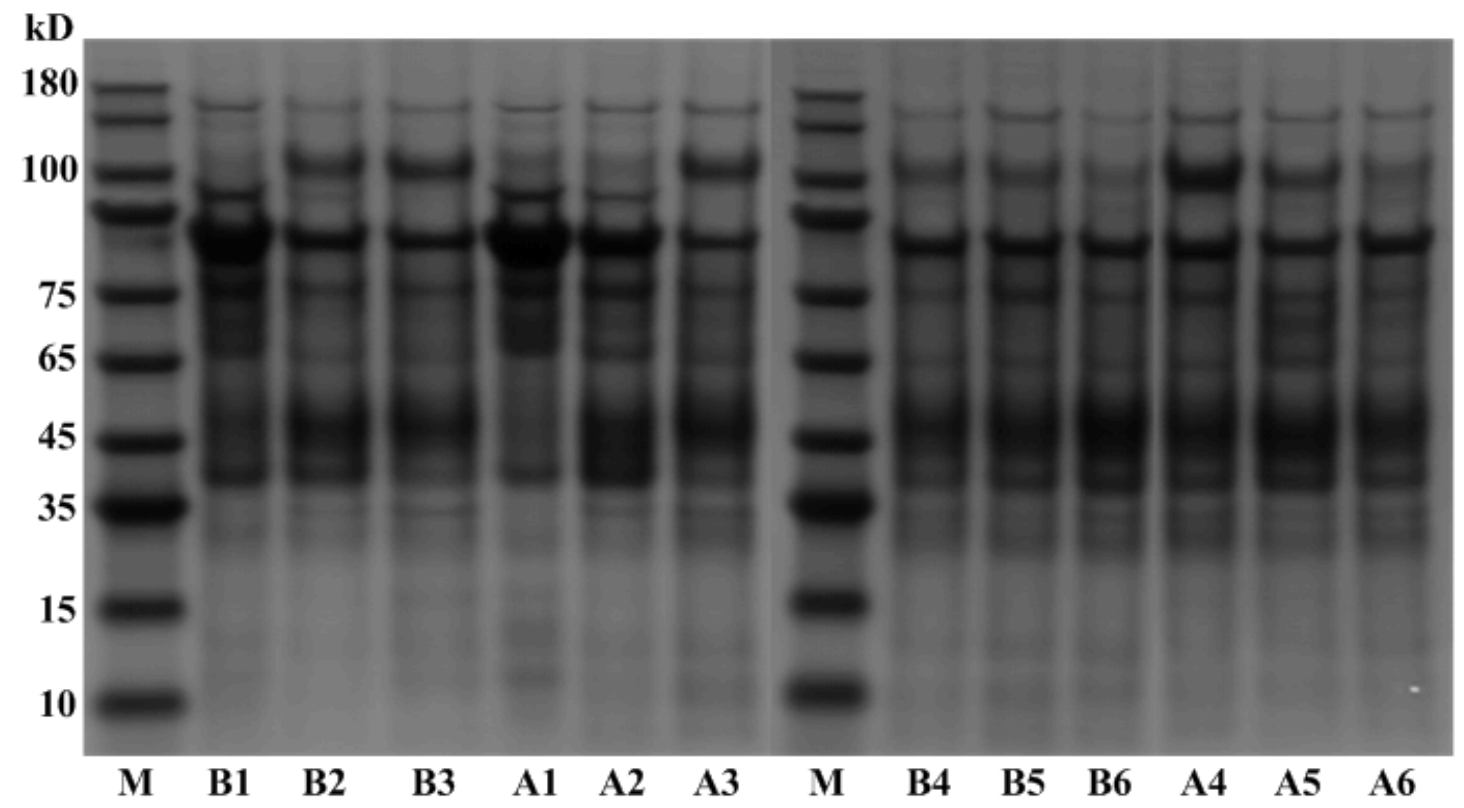

Figure 4 SDS-PAGE analysis of urinary protein samples.

M: Protein marker; BI-6: Urinary protein samples before coffee intake;A I-6: Urinary protein samples after coffee intake. 

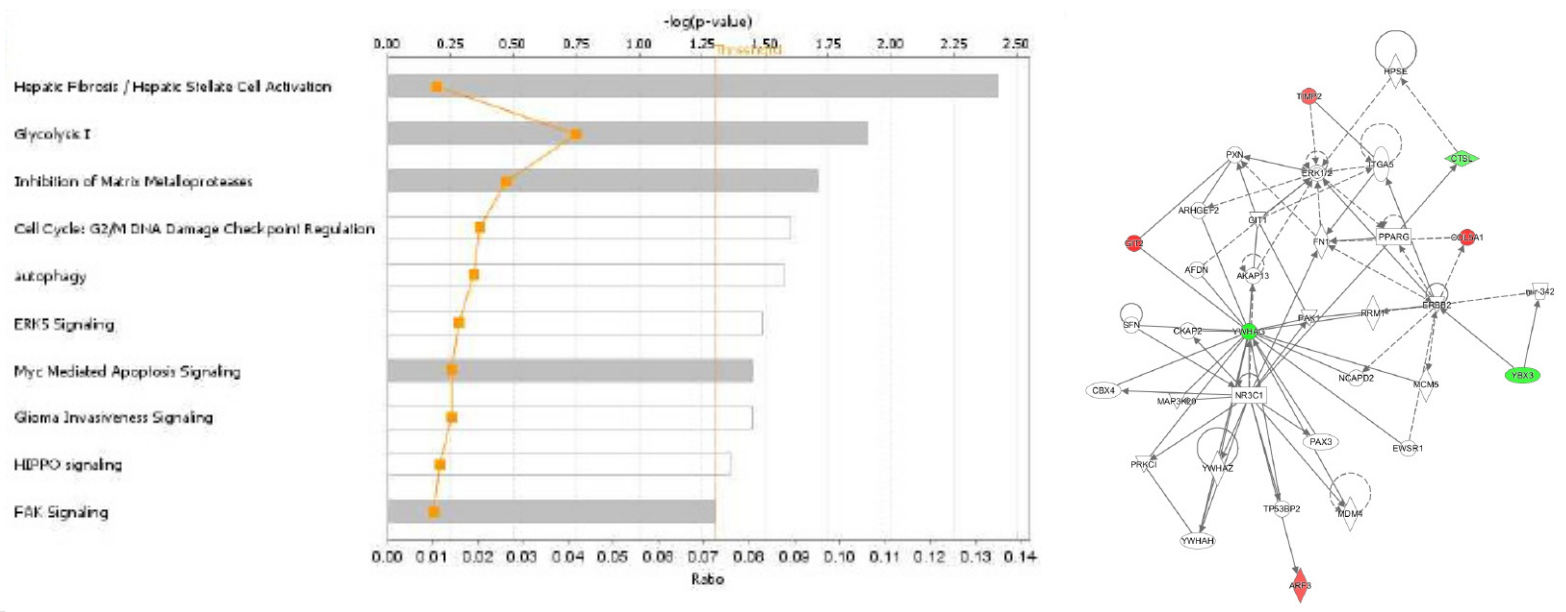

Figure 5 Functional analysis of the protein changes induced by acute coffee intake.

A. Canonical pathways. The $y$ axis shows the negative log of the p value. B. YWHAG network.

Table I Changed proteins after acute coffee intake

\begin{tabular}{|c|c|c|c|c|}
\hline Accession & Protein name & Fold change & $P$ value & Candidate biomarkers of diseases \\
\hline Q5T5J6 & Transcriptional protein SWTI & 1.89 & 0.019 & None \\
\hline B4DR69 & Neuronal PAS domain-containing protein I & 0.49 & 0.05 & None \\
\hline P077II & Cathepsin LI & 0.66 & 0.01 & None \\
\hline PI7858-2 & Isoform 2 of 6-phosphofructokinase, liver type & 0.48 & 0.046 & None \\
\hline P20908 & Collagen alpha-I(V) chain & 1.95 & 0.026 & None \\
\hline PI6989 & DNA-binding protein $\mathrm{A}$ & 0.53 & 0.041 & None \\
\hline Q14I6I-8 & Isoform 8 of ARF GTPase-activating protein GIT2 & 2.01 & 0.037 & None \\
\hline P61981 & 14-3-3 protein gamma & 0.45 & 0.041 & None \\
\hline F5GWY5 & Podocalyxin & 1.55 & 0.017 & None \\
\hline PI6035 & Metalloproteinase inhibitor 2 & 1.54 & 0.042 & Bladder cancer \\
\hline B7ZB63 & ADP-ribosylation factor 3 & 1.6 & 0.043 & None \\
\hline
\end{tabular}

The networks analysis showed that the cellular movement, growth and proliferation network were mostly affected (Figure 5B). YWHAG which is critical for maintaining cellular homeostasis, signal transduction and various pathophysiological processes,,$^{14,15}$ is a core component of this networks. In this networks, ADP-ribosylation factor 3 and Isoform 8 of ARF GTPase-activating protein which involve in energy metabolism, are related to YWHAG.

Compared with the effects of prazosin, aspirin, or pentobarbital sodium on urine proteome, ${ }^{16-18}$ the protein changes in this study were substantially different. Changes in urine proteome may be caused by many different confounding factors, such as gender, ${ }^{19} \operatorname{diet}^{20}$ and exercise, ${ }^{21}$ which indicates that the regulation of urinary proteins is complicated. In order to search for potential biomarkers which are truly associated with disease states or physiological variations, analyzing the effects of confounding factors on urine proteome is necessary. Based on current identification technology and quantitation strategy, the effects of acute coffee on urine proteome may not big enough. When animal models were used, the interference of confounding factors can be minimize and the association of urinary proteins with coffee may be more directly. ${ }^{22}$ 
Table SI Proteins identified from urine

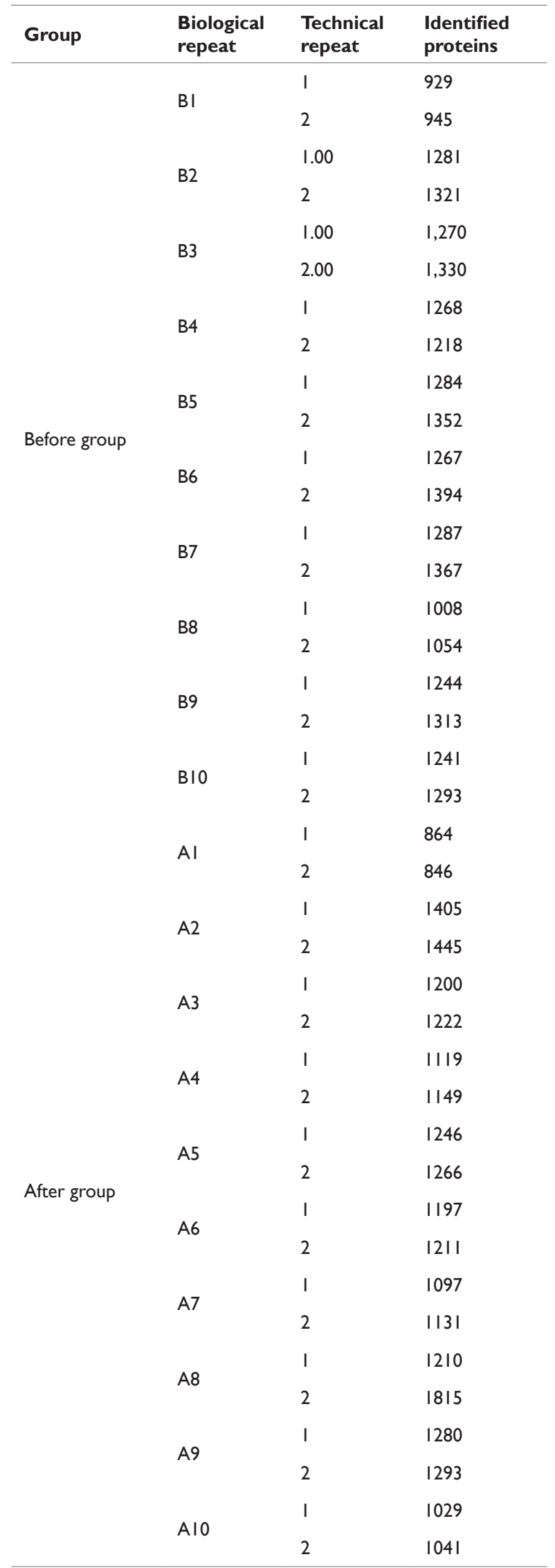

\section{Conclusion}

In this study, the effects of acute coffee intake on the urine proteome were analyzed by label-free quantitative proteomics. A total of 11 proteins were found to be significantly altered, and one protein has been previously reported as candidate disease biomarker. Based on current identification technology and quantitation strategy, acute coffee intake may be able to affect the urine proteome and disturb urinary biomarker research. Thus, when using clinical samples, the effect of coffee should be taken into consideration.

\section{Acknowledgements}

This work was supported by the National Key Research and Development Program of China (2016 YFC 1306300); the National Basic Research Program of China (2013CB530850); Beijing Natural Science Foundation $(7173264,7172076)$ and funds from Beijing Normal University (11100704, 10300-310421102).

\section{Conflict of interest}

The author declares no conflict of interest.

\section{References}

1. Gao Y. Urine-an untapped goldmine for biomarker discovery? Sci China Life Sci. 2013;56(12):1145-1146.

2. Ni Y, Gao Y. Should We Search for Early Brain Disease Biomarkers in Urine. Author Jnl. 2016;1(1):00003.

3. Airoldi L, Magagnotti C, Iannuzzi AR, et al. Effects of cigarette smoking on the human urinary proteome. Biochem Biophys Res Commun. 2009;381(3):397-402.

4. Afkarian M, Bhasin M, Dillon ST, et al. Optimizing a proteomics platform for urine biomarker discovery. Mol Cell Proteomics. 2010;9(10):2195204.

5. Wu J, Gao Y. Physiological conditions can be reflected in human urine proteome and metabolome. Expert Rev Proteomics. 2015;12(6):623-636.

6. Liu JJ, Crous-Bou M, Giovannucci E, et al. Coffee Consumption Is Positively Associated with Longer Leukocyte Telomere Length in the Nurses' Health Study. J Nutr. 2016;;146(7):1373-1378.

7. Freedman ND, Park Y, Abnet CC, et al. Association of coffee drinking with total and cause-specific mortality. N Engl J Med. 2012;366:1891904.

8. van Dam RM, Willett WC, Manson JE, et al. Coffee, caffeine, and risk of type 2 diabetes: a prospective cohort study in younger and middle-aged U.S. women. Diabetes Care. 2006;29(2):398-403.

9. Yuan $\mathrm{Y}$, Zhang $\mathrm{F}, \mathrm{Wu} \mathrm{J}$, et al. Urinary candidate biomarker discovery in a rat unilateral ureteral obstruction model. Sci Rep. 2015;5:9314.

10. Wiśniewski JR, Zougman A, Nagaraj N, et al. Universal sample preparation method for proteome analysis. Nat Methods. 2009;6(5):359 362.

11. Shao C1, Li M, Li X, et al. A tool for biomarker discovery in the urinary proteome: a manually curated human and animal urine protein biomarker database. Mol Cell Proteomics. 2011;10(11):M111.010975.

12. Eissa S, Shabayek MI, Ismail MF, et al. Diagnostic evaluation of apoptosis inhibitory gene and tissue inhibitor matrix metalloproteinase-2 in patients with bladder cancer. IUBMB Life. 2010;62(5):394-399.

13. Su Y, Gong Z, Wu Y, et al. Diagnostic Value of Urine Tissue Inhibitor of Metalloproteinase-2 and Insulin-Like Growth Factor-Binding Protein 7 for Acute Kidney Injury: A Meta-Analysis. PLoS One. 2017;12(1):e0170214. 
14. Wang Z, Nesland JM, Suo Z, et al. The prognostic value of 14-3-3 isoforms in vulvar squamous cell carcinoma cases:14-3-3beta and epsilon are independent prognostic factors for these tumors. PLoS One. 2011;6(9):e24843.

15. Zhang H, Zhang H, Lin Y, et al. RIP1-mediated AIP1 phosphorylation at a 14-3-3-binding site is critical for tumor necrosis factor-induced ASK1-JNK/p38 activation. J Biol Chem. 2007;282(20):14788-14796.

16. Zhao M, Li X, Li M, et al. Effects of anesthetics pentobarbital sodium and chloral hydrate on urine proteome. Peer J. 2015;3:e813.

17. Zhao M, Wu J, Gao Y. The Specific alpha1-Adrenergic Receptor Antagonist Prazosin Influences the Urine Proteome. PLoS One. 2016;11(10):e0164796.

18. Li X, Gao Y. Effect of Low-Dose Aspirin on Candidate Biomarkers in the Urine Proteome. MOJ Proteomics \& Bioinform. 2016;3(3):00087.
19. Liu X, Shao C, Wei L, et al. An individual urinary proteome analysis in normal human beings to define the minimal sample number to represent the normal urinary proteome. Proteome Sci. 2012;10(1):70.

20. Binder $\mathrm{H}$, Wirth $\mathrm{H}$, Arakelyan $\mathrm{A}$, et al. Time-course human urine proteomics in space-flight simulation experiments. BMC Genomics. 2014;12(15 Suppl):S2.

21. Kohler M, Franz S, Regeniter A, et al. Comparison of the urinary protein patterns of athletes by $2 \mathrm{D}$-gel electrophoresis and mass spectrometry-a pilot study. Drug Test Anal. 2009;1(8):382-386.

22. Gao Y. Roadmap to the Urine Biomarker Era. MOJ Proteomics \& Bioinform. 2014;1:00005. 